\title{
Cartilaginous metaplasia of varicose veins: a case report
}

\section{A Caggiati and $M$ Franceschini}

Department of Anatomy, Sapienza University of Rome, Via Borelli 50, 00161 Rome, Italy

\begin{abstract}
Cartilaginous metaplasia of superficial veins was found in a 64-year-old woman who underwent surgery for varicose veins. At operation, some varicose veins of the medial thigh were semi-rigid and fibroelastic to the touch. Histology revealed that half the lumen was occupied by chondroid tissue. The other half was obliterated by fibrous tissue, typical of post-thrombotic involution. Possible causes of cartilaginous metaplasia are briefly discussed.
\end{abstract}

Keywords: varicose veins; thrombosis; varicophlebitis; cartilaginous metaplasia

\section{Introduction}

It is well known that venous thrombi may undergo re-canalization or fibrous transformation. Less frequently, thrombosis may evolve in mural calcification and even ossification. In this paper, we report a case in which cartilaginous metaplasia was observed in the great saphenous vein (GSV) and its tributaries as a long-term consequence of superficial thrombophlebitis.

\section{Report}

On 23 November 2009, SC, a 64-year-old Caucasian woman, underwent intervention for varicose veins in the right leg. Her medical history included a long-lasting history of primary varicose veins (35 years) and an episode of superficial thrombophlebitis in the right thigh in 1998 treated with compression and heparin.

Preoperative duplex ultrasound demonstrated obliteration of the right GSV from the upper leg to the mid-thigh, and of the thigh portion of its anterior accessory. The lumen was occupied by inhomogeneous material showing partial re-canalization (Figure 1a). A small artery originating from periad-

Correspondence: Alberto Caggiati MD PhD, Department of Anatomy, Sapienza University of Rome, Via Borelli 50, 00161 Rome, Italy.

Email: alberto.caggiati@uniroma1.it

Accepted 26 September 2011
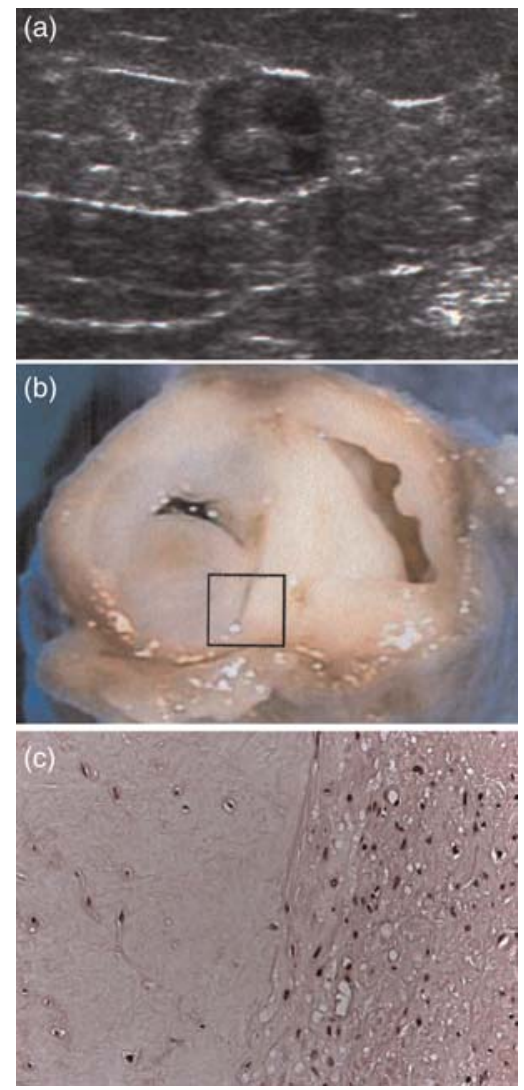

Figure 1 Anterior accessory great saphenous vein. (a) Preoperatory duplex appearance. (b) Stereomicroscopy of the lumen. The box indicates the area observed by light microscopy in (c). (c) The different tissues occupying the lumen as evidenced by light microscopy (haematoxylin-eosin, original magnification $\times 20$ ) 

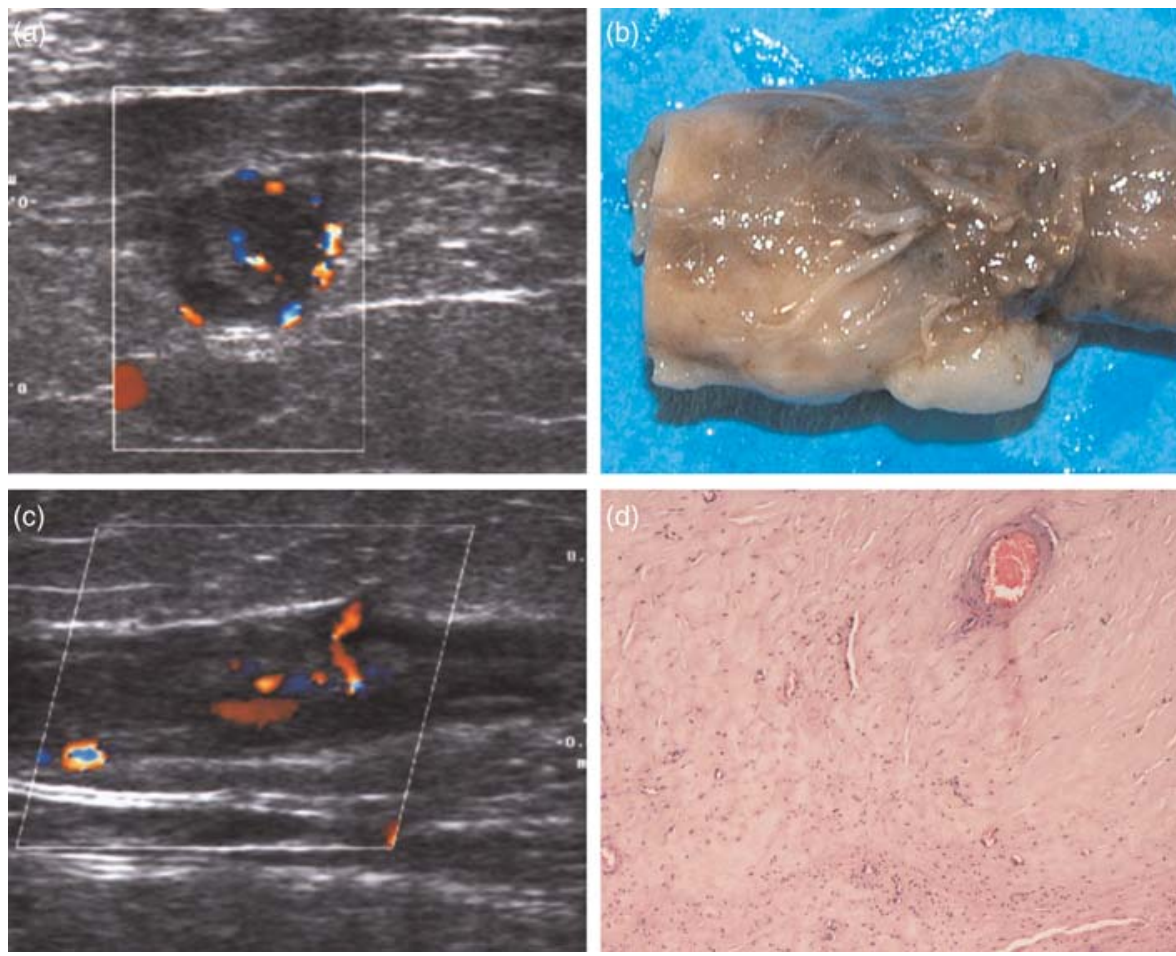

Figure 2 Great saphenous vein. The rich net of periadventital vessels demonstrated by duplex (a) and stereomicroscopy (b). A small artery running in the middle of the obstructed vessel as seen by duplex (c) and demonstrated by histology (d)

ventitial vessels (Figure 2a) ran in the middle of the lumen (Figure 2b). No pain was triggered by compressive manoeuvres with probes. Clinical and laboratory investigations did not reveal active venous inflammation or thrombosis.

At operation, the occluded varicose veins were semi-rigid and elastic to the touch, as if a silicon probe would lie inside. Therefore, samples from the anterior accessory were avulsed for histological diagnosis. Due to the hardness of the material that occupied the lumen, large scissors were necessary to cut the vein.

A cylindrical biopsy, $4.5 \mathrm{~cm}$ in length and about $10 \mathrm{~mm}$ in diameter, underwent laboratory investigation.

At stereomicroscopy, the outer surface showed large periadventitial tortuous vessels (Figure 2c). About one half of the lumen was occluded by translucent material. The other half was occupied by greyish fibrous-like tissue. Irregular fissures were in both parts of the lumen (Figure 1b).

The biopsy was then cut into smaller specimens to be investigated microscopically. Besides routine haematoxylin-eosin, alcian blue and toluidine blue stains were applied for the demonstration of cartilage cells and their specific matrix proteins.

One half of the lumen was obliterated by typical fibrous tissue, rich in fibroblasts (Figure 1c).
The other part of the lumen was obliterated by dense connective tissue in which uninuclear chondrocytes were visible within lacunae, surrounded by a basophilic matrix (Figures 1c and 3). Toluidine blue and alcian blue stains confirmed the presence of chondroid tissue (Figure 3). Irregularly shaped lumens were lined by endothelium. A small artery ran in the middle of the occluded lumen (Figure 2d). No significant infiltration, calcification and ossification were observed.

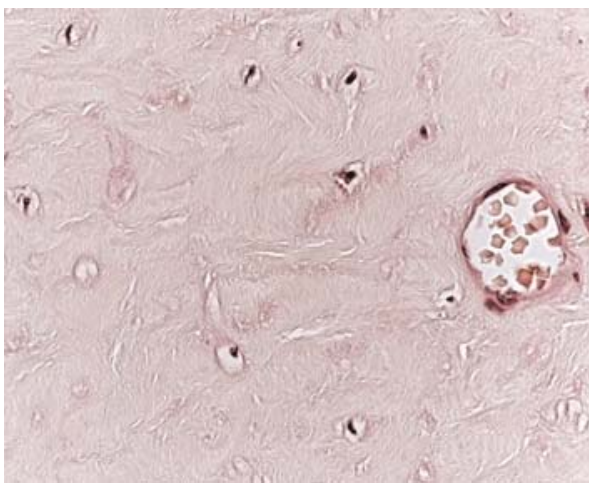

Figure 3 Uninuclear chondrocytes lie into lacunae surrounded by a basophilic matrix and small vessels (alcian blue, original magnification $\times 20$ ) 
No complications were noted until December 2010. Postoperative clinical and laboratory investigations were negative for neoplasia, hypercoagulability, autoimmune diseases or dysmetabolic disorders.

\section{Discussion}

The fate of venous thrombi is to dissolve or to transform in fibrous tissue. Venous calcification may occur also, if less frequently. To the best of a thorough revision of the literature, this report is the first to demonstrate a 'chondroid transformation' of a peripheral vein due to cartilaginous metaplasia of a thrombus. Chondroid transformation of the venous wall has been described in relation to the presence of a central catheter or renal dysfunction in humans, ${ }^{1}$ or to bypass procedures in the $\operatorname{dog}^{2}{ }^{2}$ Cartilaginous metaplasia is more frequent in arteries where it is associated with, but not causative for atherosclerotic vessel degeneration and calcification. ${ }^{3}$

On the basis of our findings, no hypothesis can be done on the pathogenesis of the changes observed. Clinical and laboratory investigations were negative for other diseases possibly determining the changes observed. As a consequence, chondroid transformation should be considered a non-specific response to tissue injury following thrombophlebitis. As in the case of arterial calcification, this process could be related to the expression of growth factors, matrix proteins and other bone-related proteins. An important role could be played by the cytoskeletal gene SM22 $\alpha$, which has recently been demonstrated to intervene in prochondrogenesis of the arterial media in response to injury. ${ }^{4}$ In particular, cartilaginous metaplasia could be due to transformation of $\alpha$-smooth muscle cells by the expression of transforming growth factor $\beta 1 .{ }^{5}$

\section{References}

1 Wali MA, Eid RA, Dewan M, Al-Homrany MA. Preexisting histopathological changes in the cephalic vein of renal failure patients before arterio-venous fistula (AVF) construction. Ann Thorac Cardiovasc Surg 2006;12: $341-8$

2 Shen J, Yang M, Jiang $\mathrm{H}$, et al. Arterial injury promotes medial chondrogenesis in Sm22 knockout mice. Cardiovasc Res 2011;90:28-37

3 Aigner T, Neureiter D, Campean V, Soder S, Amann K. Expression of cartilage-specific markers in calcified and non-calcified atherosclerotic lesions. Atherosclerosis 2008; 196:37-41

4 Speer MY. Smooth muscle cells in pathogenesis of vascular medial cartilaginous metaplasia. Cardiovasc Res 2011; 90:1-2

5 Mathieu P, Roussel JC, Dagenais F, Anegon I. Cartilaginous metaplasia and calcification in aortic allograft is associated with transforming growth factor beta 1 expression. J Thorac Cardiovasc Surg 2003;126:1449-54 